\title{
Commerce en ligne du lait de chamelle : nouveaux acteurs, nouveaux marchés
}

\author{
Gaukhar Konuspayeva ${ }^{1,2 *}$ Bernard Faye ${ }^{1,2}$ \\ Guillaume Duteurtre ${ }^{2}$
}

\section{Mots-clés}

Lait de chamelle consommation, exportation, commerce international, commerce électronique

(c) G. Konuspayeva et al., 2021

\section{(ब) (1)}

https://creativecommons.org/licenses/by/4.0/

Submitted: 4 December 2020

Accepted: 25 May 2021

Published: 30 September 2021

DOI: 10.19182/remvt.36746

\section{Résumé}

La croissance rapide du cheptel mondial de dromadaires et de chameaux vise notamment à répondre à une demande croissante en lait de chamelle. Nous analysons ces mutations en étudiant le commerce en ligne, apparu récemment, de produits issus de lait de chamelle. Cette étude souligne l'émergence d'acteurs investis dans la production intensive de lait de chamelle, mais aussi dans le commerce de lait en poudre, de lait fermenté et de fromages. Ces nouveaux entrepreneurs du lait de chamelle sont implantés pour une bonne partie dans des pays dépourvus d'élevages camelins, notamment en Europe, aux Etats-Unis, au Canada et dans plusieurs pays émergents. Les prix du lait vendu par internet sont extrêmement variables, soulignant que ce marché est en cours de construction. L'émergence de ce commerce reflète une dynamique d'innovation duale. A cette économie laitière entrepreneuriale basée sur des échanges entre des fermes d'élevage intensif et des consommateurs éloignés s'oppose en effet une économie laitière de proximité basée sur le commerce périurbain de lait frais fortement corrélé aux systèmes pastoraux. Nous concluons sur des pistes de recherche et de développement pour rendre le commerce numérique de lait de chamelle plus inclusif.

- Comment citer cet article : Konuspayeva G., Faye B., Duteurtre G., 2021. Online camel milk trade: new players, new markets. Rev. Elev. Med. Vet. Pays Trop., 74 (3): 137-144, doi: 10.19182/remvt.36746

\section{INTRODUCTION}

On assiste depuis un peu plus de deux décennies à un intérêt croissant pour les productions camelines, qui se traduit notamment par une croissance rapide du troupeau de camelins dans plusieurs pays. Le taux de croissance du cheptel camelin mondial dépasse ainsi celui des autres espèces d'herbivores domestiques à l'exception de la chèvre (Faye, 2020). Certains évoquent même un véritable enthousiasme pour les produits issus de l'élevage camelin (Faye, 2018a) qu'on peut associer à trois principaux facteurs. En premier lieu, les changements climatiques qui se traduisent par une augmentation des irrégularités pluviométriques et par l'aridification de certains milieux ont généré une expansion des zones d'élevage des camelins notamment dans les régions semi-arides d'Afrique (Faye et al., 2012). En second lieu, l'insertion croissante des élevages dans l'économie marchande a permis l'essor progressif du commerce des produits camelins et

1. Al-Farabi National Kazakh University, Almaty, Kazakhstan. Kazakh Research Institute of Livestock and Fodder Production, 51 Zhandosov str., 050035, Almaty, Kazakhstan.

2. CIRAD, UMR SELMET, F-34398 Montpellier, France.

SELMET, Univ Montpellier, CIRAD, INRAE, Institut Agro, Montpellier, France.

* Autrice pour la correspondance

Email : konuspayevags@hotmail.fr notamment du lait de chamelle. Cette marchandisation des produits camelins s'est appuyée sur le développement de systèmes de collecte du lait et d'unités de transformation qui commercialisent divers types de produits laitiers sur les marchés locaux, régionaux, voire internationaux (Faye, 2016). Enfin, en troisième lieu, on prête aux produits camelins (lait, viande ou laine) des vertus diététiques et médicinales, réelles ou supposées, qui motivent leur consommation pour des usages alimentaires et non alimentaires (cosmétiques, vêtements) (Yadav et al., 2015).

Pour comprendre la dynamique de ce nouvel intérêt pour l'élevage camelin et ses produits, le présent article se focalise sur le commerce en ligne du lait de chamelle. La consommation de ce lait connaît en effet un engouement sans précédent dans de nombreux pays. Alors qu'elle était restée limitée jusqu'à récemment à l'autoconsommation dans les élevages, on observe aujourd'hui une augmentation rapide des ventes à destination des zones urbaines (Sraïri et al., 2019). Or, du fait de l'éloignement des consommateurs des zones de production, ces acteurs de ce nouveau commerce se sont engagés depuis plusieurs années dans le recours à la vente par internet. Après avoir rappelé les évolutions de la production et de la consommation mondiale du lait de chamelle, nous analysons ces innovations en proposant une description précise des différents produits commercialisés, de leurs conditions de production, ainsi qu'une étude systématique des sites web engagés dans ce commerce en ligne. 


\section{LAIT DE CHAMELLE : UNE PRODUCTION EN PLEINE EXPANSION}

La production effective de lait de chamelle à l'échelle mondiale est très mal renseignée pour de multiples raisons : faible intégration au marché jusqu'à une époque récente, éloignement des bassins de production avec difficulté d'accès aux bassins de consommation, faible part des produits transformés et surtout importance de l'autoconsommation. La part autoconsommée est en effet largement prépondérante bien que difficile à chiffrer. Même dans un pays où la filière connaît une certaine structuration (Arabie Saoudite), la part du lait produit dans le système bédouin totalement non intégré au marché laitier représentait, selon une estimation faite dans le nord du pays, $62 \%$ du lait de chamelle et, sur l'échantillon de fermes suivies dans cette étude, $13 \%$ seulement approvisionnaient le marché (Faye et al., 2014a). La part du lait autoconsommée est probablement plus élevée dans les pays où les zones d'élevage camelin sont très éloignées des centres urbains (pays sahéliens par exemple) et elle l'est moins dans les pays de taille modeste dotés de bonnes infrastructures routières et logistiques (pays du Golfe notamment). Dans tous les cas, malgré le manque de données, l'autoconsommation de lait de chamelle est largement répandue. Les systèmes d'élevages extensifs, pastoraux, se prêtent en effet mal à la valorisation économique du lait de chamelle.

Les statistiques officielles de la FAO affichent une production mondiale de 3,1 millions de tonnes de lait en 2018, ce qui, compte tenu de ce qui est rapporté ci-dessus, apparait largement sous-estimé. En s'appuyant sur la seule référence $\mathrm{FAO}$, le lait de chamelle ne représenterait que $0,37 \%$ du lait consommé dans le monde, ce qui en fait un produit malgré tout marginal, même si cette proportion a doublé depuis 1961 $(0,18 \%)$. Par ailleurs, avec une proportion de $23 \%$ des effectifs dévolus à la production laitière, la chamelle apparait plus largement élevée

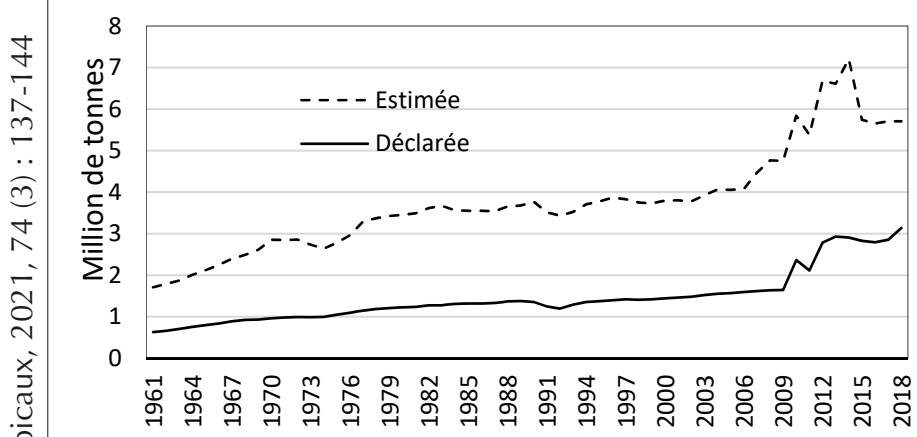

Figure 1 : Production de lait de chamelle $(\times 1000$ t) dans les 48 pays déclarant officiellement un cheptel camelin dans la base FAOstat, 1961-2018 /// Camel milk production ( $\times 1000 t$ ) in the 48 countries officially reporting camel livestock in FAOstat database, 1961-2018 pour ce type de spéculation que la vache dont $18 \%$ seulement des individus sont consacrés à la production de lait (FAOstat, 2021). En se référant à cette proportion et compte tenu des effectifs mondiaux de grands camélidés estimés à 35 millions de têtes (FAOstat, 2021 ; Faye, 2021), il y aurait un peu plus de huit millions de chamelles laitières dans le monde dont environ la moitié (quatre millions) seraient en lactation, le cycle de la chamelle étant de deux années en moyenne. En se basant sur une moyenne d'environ 1500 litres de lait par lactation (Faye, 2004 ; Abdalla et al., 2015) soit approximativement quatre litres par jour, on peut estimer la production mondiale annuelle à six millions de tonnes de lait de chamelle (figure 1).

Ces estimations sont encore loin des quantités produites par les autres espèces laitières à l'échelle de la planète. Cependant, si l'on se réfère aux seuls pays déclarant officiellement un cheptel camelin (48 pays répertoriés), la part de la production de lait de chamelle est plus importante que celle du lait de brebis. La part du lait de chamelle dans la production par pays varie de $0,001 \%$ (Russie) à 44,6 \% (Somalie), voire $60 \%$ au Sahara occidental, zone incluse depuis 2019 par la FAO dans les provinces sahariennes du Maroc (figure 2).

Au total, la production de lait de chamelle dans le monde estimée par la FAO a été multipliée par cinq depuis 1962. Cette envolée correspond à un taux de croissance annuel de $7 \%$, soit plus du double du taux de croissance du lait de vache, et plus du triple du taux de croissance du lait de petits ruminants (FAOstat, 2021).

L'un des changements majeurs de ces dernières décennies en matière de production laitière de lait de chamelle est l'émergence de systèmes d'élevage laitier intensifs caractérisés par un arrêt de la mobilité des troupeaux (Chamekh et al., 2020). Les pays du Golfe, et les pays d'Asie centrale à l'époque soviétique ont été pionniers en la matière. L'intensification laitière dans la filière cameline consiste à considérer la possibilité d'élever les chamelles en production comme des vaches laitières en mobilisant toutes les pratiques qui ont conduit à un développement laitier sans précédent dans le monde occidental : usage des biotechnologies de la reproduction (insémination artificielle, transfert d'embryons), traite mécanique, prévention sanitaire, alimentation raisonnée, sédentarité, contrôle des performances et sélection génétique (Faye, 2018a ; 2018c).

Cependant, si le potentiel de production de la chamelle peut être aisément comparé à celui des zébus élevés en zone tropicale, il apparait plus faible par rapport aux vaches laitières sélectionnées, en raison d'un cycle de reproduction deux fois plus long, de l'inadaptation des machines à traire utilisées en élevage bovin, et des besoins alimentaires plus spécifiques (Nagy et al., 2015 ; Ayadi et al., 2018 ; Faye, 2016). De ce fait, les coûts de production du lait de chamelle en fermes intensives sont élevés et le résultat économique d'une ferme cameline intensive ne peut être bénéfique qu'avec un prix de vente du lait lui aussi élevé. C'est pour l'instant largement le cas puisque dans

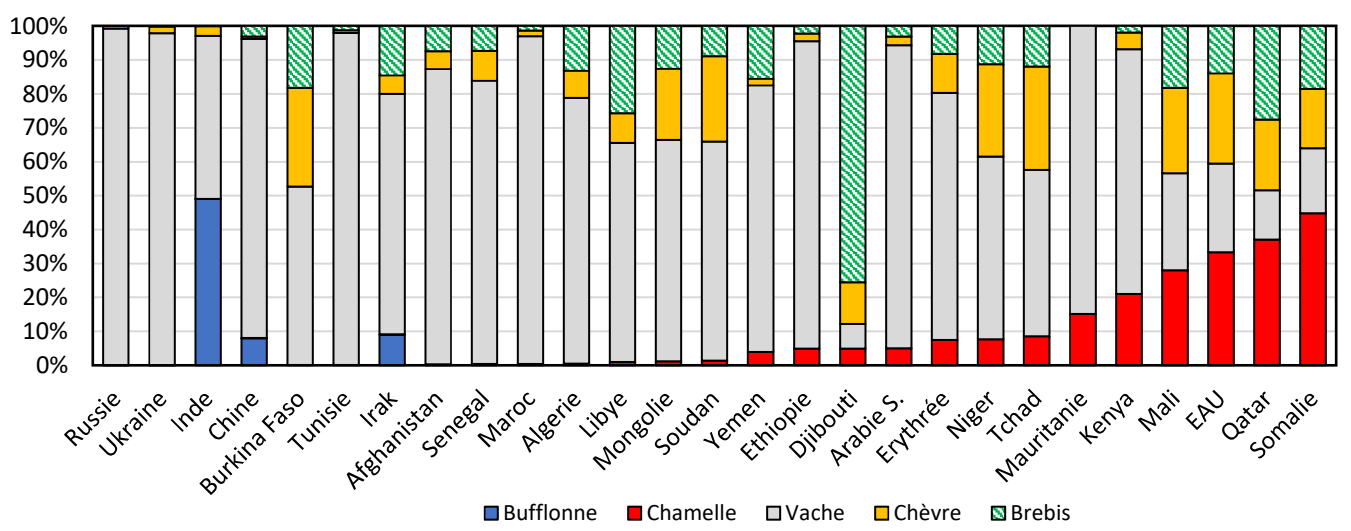

Figure 2 : Proportions des laits des différentes espèces dans les 28 pays déclarant une production nationale de lait de chamelle (calculées d'après les données de productivité estimées ou declarées par pays en 2019, FAOstat, 2021) /// Proportions of milk from different species in the 28 countries reporting national camel milk production (calculated from estimated or reported productivity data by country in 2019, FAOstat, 2021) 
la plupart des pays producteurs, le lait de chamelle est commercialisé à un prix deux à trois fois, voire plus, supérieur à celui du lait de vache (Faye et al., 2014b). En l'absence d'une réelle sélection génétique et d'un développement des biotechnologies de la reproduction en dehors des stations de recherche (Faye, 2018b), l'augmentation des performances laitières restera limitée au choix des quelques meilleures laitières dans des fermes intensives en dépit des récents progrès sur la connaissance du potentiel génétique de l'espèce (Al-Abri et Faye, 2019 ; Burger et al., 2019).

\section{CONSOMMATION DANS LE MONDE}

La consommation de lait de chamelle par habitant et par an est difficile à estimer compte tenu de la part considérable de lait autoconsommé chez les populations pastorales. Par exemple dans une enquête réalisée dans la périphérie de N’Djamena (Tchad), Koussou (2008) rapporte que la part autoconsommée de lait de chamelle varie de 1,7 à $30 \%$ du lait disponible selon les ménages avec une moyenne de 7,4 \%. Il s'agit de ménages établis de manière saisonnière autour de la capitale et, de ce fait, intégrés au marché urbain. L'autoconsommation y est en proportion largement plus importante dans les zones éloignées des bassins de consommation.

En première estimation et sur la base de la population humaine enregistrée dans FAOstat, la consommation dans les pays producteurs de lait de chamelle aurait varié de 0,40 litre/hab./an en 1961 à 0,81 litre/ hab./an en 2018, soit une légère augmentation avec des résultats très contrastés selon les pays. Dans la plupart des pays concernés, et pas seulement dans les pays très peuplés évoqués ci-dessus, la consommation est souvent limitée à certaines régions. C'est le cas notamment des pays du Maghreb où le lait de chamelle est un produit des régions du Sud, mais aussi dans certains pays sahéliens où le lait de chamelle est consommé uniquement ou presque dans les zones sahariennes (Mali, Niger) ou dans les basses-terres arides (Ethiopie, Kenya). En conséquence, calculer une consommation moyenne par habitant en tenant compte de l'ensemble de la population sous-estime grandement la place du lait de chamelle dans la consommation des ménages de zones arides, d'autant plus que la croissance démographique humaine y est moindre. On ne dispose malheureusement que de peu de données sur le sujet. Par exemple dans les zones pastorales du Kenya, une étude portant sur 202 ménages montre que le lait de chamelle représente en moyenne $20 \%$ environ du total des dépenses alimentaires du ménage, sachant que plus de $60 \%$ des ménages déclarent en consommer (Elhadi et al., 2015). Une variabilité saisonnière importante est observée, le pourcentage des dépenses en lait de chamelle passant de $14 \%$ en saison des pluies à $28 \%$ en saison sèche. En quantité, cela représente 2 litres/jour/ménage en saison des pluies et 2,5 litres en saison sèche, soit à l'échelle de l'année, plus de deux fois plus que de lait de vache. La taille moyenne du ménage étant de six personnes, on arrive à une consommation de l'ordre de 135 litres/hab./an, soit des proportions nettement plus importantes que les moyennes nationales.

Par ailleurs, il convient de noter que la consommation de lait de chamelle ne se limite pas aux pays producteurs mais elle est présente également dans les pays du Nord. Un commerce se met petit à petit en place, même s'il n'apparait ni dans les données statistiques des douanes ni dans les données de la FAO. Il y a quelques années, on pouvait en effet écrire que le lait de chamelle n'était l'objet que d'un marché local, voire national, car il n'existait pas de marché international (Faye et Konuspayeva, 2012). Ce n'est plus vrai aujourd'hui. La marchandisation du lait de chamelle évoquée plus haut a en effet permis à ce produit de se diffuser à une plus large échelle, notamment en milieu urbain, soit dans des circuits informels par la vente directe du lait provenant d'élevages périurbains (Koussou et al., 2012), soit dans des circuits formels avec transformation en laiteries et vente dans des réseaux de distributeurs incluant les grandes surfaces. De fait, la mise sur le marché du lait de chamelle s'est traduite par deux changements importants, la diversification des produits proposés et l'internationalisation de sa vente. La diversification des produits sera abordée dans une autre publication. Dans la présente étude, nous nous sommes intéressés aux ventes des produits laitiers camelins par internet.

\section{COMMERCE EN LIGNE}

Longtemps réservé au don à l'hôte de passage ou aux besoins de la famille élargie ou du campement, le lait de chamelle est resté pendant longtemps en dehors des transactions commerciales. Dans de nombreuses sociétés pastorales, le tabou de la vente a été longtemps un frein à l'émergence d'un commerce local (Abeiderrahmane, 1997). Les mutations en cours tant sur le plan des systèmes de production camelin (Faye, 2018c) que sur le plan de de l'urbanisation ont conduit à l'émergence d'un marché du lait de chamelle (Faye et Konuspayeva, 2017). D'abord limité à des ventes locales ce commerce a connu récemment une internationalisation rendue possible par l'apparition du lait de chamelle en poudre. Ce commerce de longue distance a tout naturellement profité de l'essor des nouvelles possibilités offertes par le commerce en ligne.

En 2020, c'est-à-dire au moment de l'étude, il existait deux grandes plateformes de revente de lait de chamelle en poudre (Ali-Baba et Amazon) ainsi qu'un grand nombre d'autres sites de revente spécialisés. La plateforme chinoise Ali-Baba propose une très large gamme dont on trouvera quelques fournisseurs-revendeurs et prix du lait de chamelle en poudre dans le tableau I sachant qu'il est loin d'être exhaustif (33 parmi 138 répertoriés sur le site Ali-Baba).

On voit que les prix ramenés au kilogramme sont très variables. En effet, ramenés au kilogramme de poudre, les prix varient de 0,1 à 330 USD avec une moyenne autour de 1 USD/kg acheté à la tonne. Une telle variabilité est à mettre sur le compte a) des unités de vente (l'unité d'emballage pour la vente en gros du lait en vrac est en général constituée de sacs de $25 \mathrm{~kg}$, mais le conditionnement est également très variable pour la vente en détail, comme le montrent les différentes origines des fournisseurs ; figure 3), et b) de la qualité du produit vendu et de la fiabilité de ses origines.

A noter que les fournisseurs ne sont pas nécessairement les producteurs, la plupart d'entre eux n'étant que des revendeurs. Les sites de vente en ligne spécialisés proposent des produits plus diversifiés dans des unités de plus faibles volumes. C'est le cas du site Desert Farms aux Etats-Unis qui propose des produits issus des fermes de chamelles laitières américaines et européennes (tableau II).

En France, le site www.camel-idee.com/ propose également une gamme de produits comme alicaments à des prix plus élevés que les concurrents (tableau III), l'origine du lait provenant essentiellement de la ferme Camelicious à Dubaï.

Aux Pays-Bas, la ferme de chamelles laitières de M. Smits propose aussi sur leur site de e-commerce www.oasismilk.com/fr/camel-milkpowder-and-capsules/ des produits à base de lait de chamelle provenant de leur exploitation sur le réseau européen (tableau IV).

A Paris, la société Chamelait vend également du lait de chamelle en poudre par paquet de $100 \mathrm{~g}$ à 19,99 EUR, soit près de $200 \mathrm{EUR} /$ $\mathrm{kg}$ (https://fr-fr.facebook.com/chamelait). Le Canada et l'Inde comprennent le plus grand nombre de fournisseurs de lait de chamelle en poudre avec respectivement 26 et 20 entreprises impliquées. Parmi les autres fournisseurs, le plus grand nombre d'entreprises se situent en Allemagne, Thaillande, Autriche et Hongrie.

On constate qu'à l'exception de l'Inde, de la Chine, des Emirats Arabes Unis, du Kazakhstan et de l'Australie, la plupart des fournisseurs-revendeurs se situent dans des pays dépourvus d'élevage 
Tableau I : Liste de quelques fournisseurs de lait de chamelle sur la plate-forme de vente Ali-Baba (Chine) (www.alibaba.com/trade/) /// List of some camel milk suppliers on Ali-Baba sales platform (China) (www.alibaba.com/trade/)

\section{Fournisseur}

Pays

ype de packaging

Shree Khrishna Enterprise Inde

Shop Globally LLC

Eurasia Invest Ltd LLP

Camel'Idée

Xi'an Silk Road Dairy

Co Ltd

Tristan Morris

Avenorte Avicola Ltda

\section{Georgie}

Kazakhstan

France

Chine

Canada

Brésil

Royaume-Uni

Europe Distributor Pty Ltd Finlande

Refiwe Imp.\& Exp.

Spinel Co.

Agrosul Agroavicola Ind.

Sam speed Imp.Exp. Sasu

Eldos Hijos Food House

SARL De Canolle

Safeway Agro LLC

Morgan's Choice

Rotur Lawncare Service

Trilium group

United SA Agri supplied

Globe Exp Imp Pty Ltd

HBK90 Ltd

Global Trade Impex Ltd

Donovan Exp. Co. Ltd

Carl Marketing

Baba Thai Group

Tatian NSAN

C\&M International LLC

MHS TJYSK Group LLC
INT Exporter Pty Ltd

$\forall$ Wamenya Trading
Afrique du Sud

Thaïlande

Brésil

France

Philippines

France

Ukraine

Canada

Canada

Canada

Afrique du Sud

Allemagne

Royaume-Uni

Royaume-Uni

Thaïlande

Pays-Bas

Canada

Thaïlande

Thaïlande

Etats-Unis

Etats-Unis

$1 \mathrm{~kg} \quad 10-15 / \mathrm{kg}$

$200 \mathrm{~g}$

$1 \mathrm{~kg}$

$100 \mathrm{~g}$

$330 \mathrm{~g}$

$25 \mathrm{~kg}$

$25 \mathrm{~kg}$

$25 \mathrm{~kg}$

$200 \mathrm{~g}$

$25 \mathrm{~kg}$

$25 \mathrm{~kg}$

$24 \mathrm{~kg}$

$25 \mathrm{~kg}$

$25 \mathrm{~kg}$

$25 \mathrm{~kg}$

$25 \mathrm{~kg}$

$50 \mathrm{~kg}$

$25 \mathrm{~kg}$

$25 \mathrm{~kg}$

$25 \mathrm{~kg}$

$25 \mathrm{~kg}$

$25 \mathrm{~kg}$

$25 \mathrm{~kg}$

$2 \mathrm{~kg}$

$25 \mathrm{~kg}$

$20 \mathrm{~kg}$

$2 \mathrm{~kg}$

$25 \mathrm{~kg}$

$5 \mathrm{~kg} \quad 1350-1450 / \mathrm{t}$

$6 \mathrm{~kg} \quad 1350-1400 / \mathrm{t}$
Tableau II : Liste des produits alimentaires à base de lait de chamelle proposé par le site https://desertfarms.com/collections/camelmilk // List of food products made from camel milk available at https:// desertfarms.com/collections/camelmilk

\begin{tabular}{|c|c|c|c|c|}
\hline Produit & Unité & $\begin{array}{l}\text { Prix } \\
\text { (USD) }\end{array}$ & $\begin{array}{c}\text { Prix } \\
\text { (USD/kg) }\end{array}$ & $\begin{array}{c}\text { Prix } \\
\text { (USD/kg EL*) }\end{array}$ \\
\hline Lait frais & $16 \mathrm{oz}$ & 18 & 40,5 & 40,5 \\
\hline Lait frais & $8 \mathrm{oz}$ & 10 & 45 & 45 \\
\hline Lait congelé & $16 \mathrm{oz}$ & 18 & 40,5 & 40,5 \\
\hline Lait congelé & $8 \mathrm{oz}$ & 10 & 45 & 45 \\
\hline Kéfir congelé & $16 \mathrm{oz}$ & 17 & 38,1 & 38,1 \\
\hline Lait en poudre & $420 \mathrm{~g}$ & 120 & 285,7 & 34,4 \\
\hline Lait en poudre & $200 \mathrm{~g}$ & 74 & 375 & 45,1 \\
\hline
\end{tabular}

* EL : équivalent lait ; $1 \mathrm{~kg}$ lait en poudre $=8,3 \mathrm{~kg}$ EL /// * EL: milk equivalent; $1 \mathrm{~kg}$ milk powder $=8.3 \mathrm{~kg} \mathrm{EL}$

Tableau III : Liste des produits proposés par le site de vente en ligne Camel'Idée en France /// List of products available on the Camel'Idée online shop in France

\begin{tabular}{|c|c|c|c|c|}
\hline Produit & Unité & $\begin{array}{l}\text { Prix } \\
\text { (EUR) }\end{array}$ & $\begin{array}{c}\text { Prix } \\
\text { (EUR/kg) }\end{array}$ & $\begin{array}{c}\text { Prix } \\
\text { (EUR/kg EL*) }\end{array}$ \\
\hline Lait en poudre & $100 \mathrm{~g}$ & 19,5 & 195 & 23,40 \\
\hline $\begin{array}{l}\text { Lait cru ou } \\
\text { surgelé }\end{array}$ & $7 \times 500 \mathrm{ml}$ & 67,5 & 19,2 & 19,20 \\
\hline Lait en poudre & $5 \times 10 g$ & 12 & 240 & 28,90 \\
\hline $\begin{array}{l}\text { Lait en poudre } \\
\text { compl. }\end{array}$ & $100 \mathrm{~g}$ & 22,5 & 225 & 27,10 \\
\hline Lait pasteurisé & $235 \mathrm{ml}$ & 5 & 21,2 & 21,20 \\
\hline
\end{tabular}

$*$ EL : equivalent lait ; $1 \mathrm{~kg}$ lait en poudre = 8,3 kg EL /// * EL: milk equivalent; $1 \mathrm{~kg}$ milk powder $=8.3 \mathrm{~kg} \mathrm{EL}$

camelin (Europe, Etats-Unis). Parmi les fournisseurs de produits camelins, il existe une entreprise marocaine, Prodigia Sarl, mais elle ne commercialise que des produits cosmétiques traditionnels dont des savons au lait de chamelle.

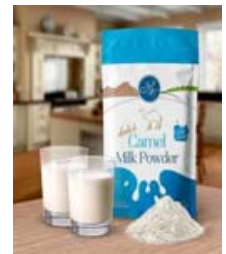

Europe Distributor (Finlande)

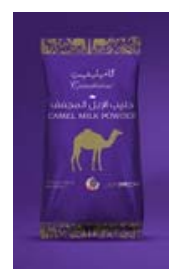

Donovan Exp. Ltd (Thaïlande)

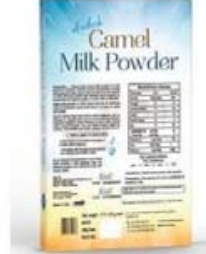

Baba Thai group (Thaïlande)

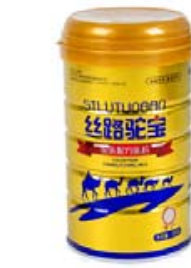

Xi'an Silk Road Co Ltd (Chine)

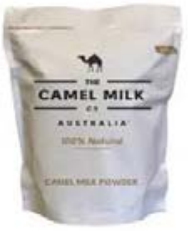

Refiwe Imp.\& Exp. (Afrique du Sud)

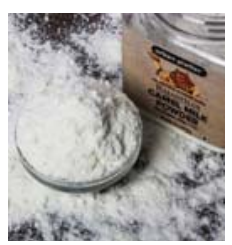

Baba Thai group (Thaïlande)

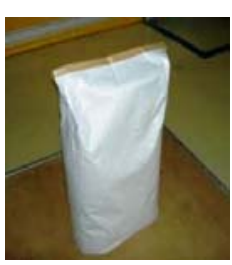

Avenorte Avicola (Brésil)

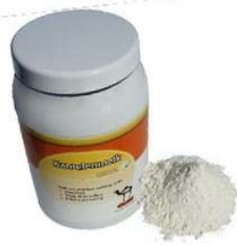

P \& K enterprise (Thaïlande)

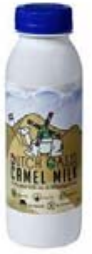

TB J Exp. Imp. Pty Ltd (Allemagne)

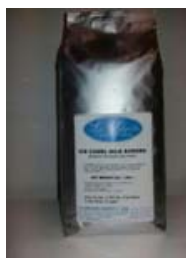

La Scintilla SAS (Italie)

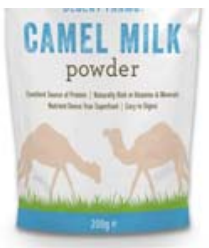

Shop Globally LLC (Georgie)

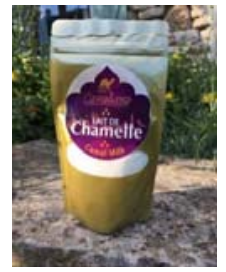

Camel'Idée (France)

Figure 3 : Quelques emballages de lait en poudre de chamelle et nom des fournisseurs sur le site de vente en ligne Ali-Baba (Chine) /// Some packages of camel milk powder and suppliers' names on Ali-Baba online sales site (China) 
Tableau IV : Liste des produits proposés par le site de vente en ligne de la ferme de M. Smits aux Pays-Bas /// List of products available on the online sales site of Mr. Smits' farm in the Netherlands

\begin{tabular}{lcccc} 
Produit & Unité & $\begin{array}{c}\text { Prix } \\
\text { (EUR) }\end{array}$ & $\begin{array}{c}\text { Prix } \\
\text { (EUR/kg) }\end{array}$ & $\begin{array}{c}\text { Prix } \\
\text { (EUR/kg EL*) }\end{array}$ \\
\hline Lait en poudre & $425 \mathrm{~g}$ & 65 & 153 & 18,40 \\
Poudre en capsule & $60 \mathrm{~g}$ & 10,5 & 175 & 21,10 \\
Lait pasteurisé & $7 \times 500 \mathrm{ml}$ & 49,5 & 14,1 & 14,1
\end{tabular}

$*$ EL : equivalent lait ; $1 \mathrm{~kg}$ lait en poudre $=8,3 \mathrm{~kg}$ EL /// $*$ EL: milk equivalent; $1 \mathrm{~kg}$ milk powder $=8.3 \mathrm{~kg} \mathrm{EL}$

Peu de fournisseurs sont susceptibles actuellement de commercialiser le lait de chamelle en poudre en Europe. Hormis le lait produit en Europe par la ferme de M. Smits aux Pays-Bas (www.kamelenmelk.nl) et les fermes américaines livrant leur produit sur le site de Desert Farms, seule la ferme Camelicious à Dubaï a pu obtenir, à notre connaissance, l'agrément de l'Union européenne pour exporter son lait vers l'Europe, à la suite d'un processus de plusieurs années impliquant non seulement la production mais aussi tous les systèmes étatiques de contrôle (Nagy et al., 2014). Si le nombre de fournisseurs-revendeurs de produits camelins est assez important à l'échelle mondiale, le nombre de producteurs-transformateurs sur le marché international apparait limité, dont nous donnons une liste assez complète ci-après.

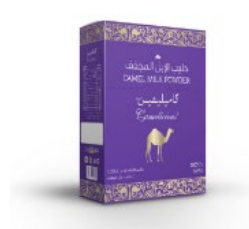

Camelicious (http://camelicious.ae/productcategory/premium-long-life-camel-milk/) : il s'agit de l'une des plus grandes fermes de chamelles laitières au monde (plus de 5000 animaux en 2018) basée à Dubaï. Démarrée en 2006, la ferme produit aujourd'hui environ deux millions de litres de lait par an. Sa production est transformée in situ en produits incluant le lait en poudre. La ferme est agréée pour l'exportation en Europe et, de fait, ses produits sont très présents sur le marché européen, mais aussi en Arabie Saoudite, aux Etats-Unis, en Russie, en Chine et en Malaisie.

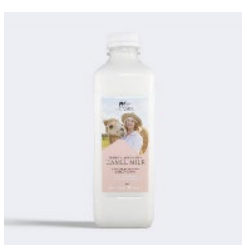

QCamel (https://qcamel.com.au/) : ce fournisseur australien situé dans le Queensland dispose d'une ferme de chamelles gérée par la famille Brisbane, fondatrice de l'Australian Camel Industry Association (ACIA). Commencée en 2006, la ferme laitière a ensuite développé sa propre laiterie. Accréditée en 2014, la laiterie a produit le premier lait de chamelle pasteurisé en Australie. Aujourd'hui, QCamel produit également des smoothies au yaourt, en passant par les poudres, les bipoudres et les cosmétiques. Ces produits sont vendus auprès de plus de 80 détaillants à travers l'Australie et exportés à l'échelle internationale vers la Nouvelle-Zélande et Singapour.

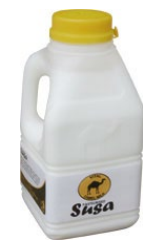

Vital Camel Milk (www.vitalcamelmilk.com/) : il s'agit de l'un des rares fournisseurs africains et de l'une des toutes premières laiteries de lait de chamelle (à l'exception de Tiviski en Mauritanie). La laiterie a démarré en effet ses opérations de transformation à Nanyuki, au Kenya, en juin 2005. Elle produit du lait pasteurisé, fermenté (susa), des yaourts à boire et des crèmes glacées. Les produits sont disponibles en supermarché et dans les boutiques de Nairobi et des grandes villes du pays. La société exporte également en Afrique du Sud. L'industrie laitière au lait de chamelle au Kenya se caractérise par son grand dynamisme et son implication dans le développement local.
Desert Farms (https://desertfarms.com) : lancée par un Saoudien ayant émigré aux Etats-Unis, puis développée par la communauté Amish, cette initiative s'est ensuite appuyée sur la transformation $\mathrm{du}$ lait en provenance de huit fermes familiales dans différents Etats du pays (Missouri, Ohio, Indiana et Pennsylvanie). Ce fournisseur assure des ventes en ligne (voir plus haut), sur l'ensemble du continent nord-américain, de lait frais, pasteurisé, congelé ou en poudre. L'initiative regroupe aussi cinq fermes européennes (Suède, Allemagne, Pays-Bas et Suisse) qui assurent une partie de l'approvisionnement sur le continent européen. Ces fermes ne sont pas forcément productrices de lait, mais elles sont surtout des lieux de revente du lait du réseau européen et notamment de la ferme Smits aux Pays-Bas.

Aadvik Foods (https://aadvikfoods.com/) : provenant directement d'éleveurs de chameaux des Etats du Gujarat et du Rajasthan en Inde, le lait de chamelle est transformé dans cette laiterie depuis 2016. Elle commercialise auprès de plus de 200000 clients réguliers du pays. La gamme comprend du lait en poudre dans différents emballages, du lait congelé, du chocolat au lait et divers produits cosmétiques. Leur marché est surtout national mais une partie de la poudre de lait est exportée vers la Chine.

Camel Milk Co. (www.thecamelmilkco.com/all-products) : cette société américaine fondée récemment (2015) est basée dans le Colorado et vend essentiellement du lait congelé (en différents volumes) et du savon au lait de chamelle sur son site. Son réseau de vente est cependant limité au niveau national.

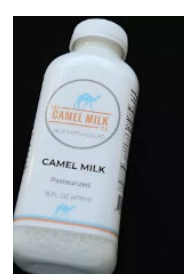

UK Camel Milk Co. (www.ukcamelmilk.co.uk/) : fondée en 2013, l'objectif principal de cette entreprise est l'approvisionnement en lait de chamelle et en produits dérivés au Royaume-Uni et dans le reste du monde. Parmi les produits proposés, on trouve le lait cru fraîchement congelé, la poudre de lait, les capsules, les savons et les crèmes. Tous ces produits proviennent en réalité de la ferme Smits aux Pays-Bas.

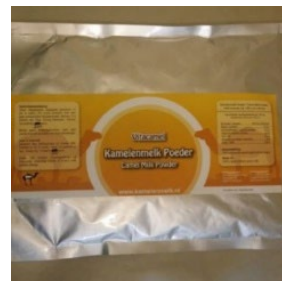

Camel Dairy Farm Smits : c'est donc une ferme laitière néerlandaise de chamelles, située dans le village de Berlicum près de Hertogenbosch. C'est la seule ferme laitière cameline d'envergure en Europe. En plus de la vente sur le marché national néerlandais, le lait de chamelle est exporté vers le RoyaumeUni (UK Camel Milk Co.), la Belgique, l'Allemagne, l'Autriche, la France et la Suède. La ferme fait partie du réseau Desert Farms. La société a été fondée par Frank Smits en 2006. La ferme laitière comprend environ 80 chamelles importées des îles Canaries dont environ 25 sont traites, fournissant environ 175 litres de lait par jour.

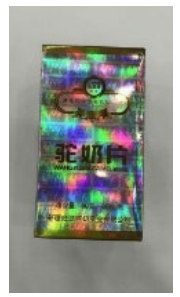

Xinjiang Wangyuan Camel Milk : cet important groupe chinois basé dans le Xinjiang propose sur le marché national une large gamme de produits alimentaires incluant des produits à base de lait de chamellelait en poudre ou liquide. Une part importante de l'activité du groupe est orientée vers la fabrication d'aliments infantiles contenant du lait de chamelle. Leurs produits sont très présents sur le marché chinois et la société représente l'un des principaux fournisseurs de la plateforme Ali-Baba. 


\section{QUELLES PERSPECTIVES DE DEVELOPPEMENT} POUR CE MARCHE ?

Il reste beaucoup d'incertitudes sur la dynamique internationale du commerce du lait de chamelle. Il y a probablement un écart important entre les volumes apparemment disponibles sur le site de vente en ligne Ali-Baba (en particulier de lait en poudre) et la réalité du nombre d'acteurs identifiés sur le marché à l'export. Une rapide estimation laisse penser que le potentiel de mise sur le marché international, sur la base des fournisseurs actuels, ne dépasserait pas 8000 tonnes de lait de chamelle par an, ce qui en théorie représente à peine $0,3 \%$ du potentiel de production mondiale. Globalement, la part de lait de chamelle disponible sur le marché à l'export est donc très faible, à comparer aux $9 \%$ du lait de vache faisant l'objet d'échanges internationaux. C'est dire la marge de progrès considérable pour ce produit, même s'il ne pourra jamais devenir un produit de substitution du lait de vache.

Ces perspectives prometteuses du commerce international du lait de chamelle sont soulignées par certains rapports d'expertises. L'un d'entre eux (Global Camel Milk Powder Market 2019 www.absolutereports.com/global-camel-milk-powder-market-14901992) évalue le taux de croissance annuel de ce marché à 3,9\% entre 2011 et 2018. Ce même rapport estime que le marché pourrait connaître une croissance annuelle de $8 \%$ pour la période 2019-2024. Dans ce contexte, la croissance comme dans bien d'autres secteurs agricoles est tirée par le marché chinois. Ce même rapport évalue le marché chinois du lait de chamelle en poudre à 99,56 millions USD en 2019 et pourrait atteindre 189,36 millions USD en 2027, soit une croissance de $8,4 \%$ à partir de 2020. L'Europe n'est cependant pas en reste. Selon la même source, la valeur du marché de la poudre de lait de chamelle aurait atteint 445,04 millions USD en 2019 et, avec une croissance annuelle de $9 \%$, pourrait atteindre 881,36 millions USD en 2027. En tête des pays européens on trouve l'Allemagne (19,0\% du marché en valeur en 2019), la France (15,9\%), la Grande-Bretagne (13,9\%), la Russie $(9 \%$ ) et l'Italie $(7 \%)$. Cette répartition serait stable sur la période 2020-2027. Les autres pays européens impliqués dans ce marché sont par ordre d'importance l'Autriche, la Pologne, le Portugal, l'Espagne, la Turquie, les Pays-Bas, l'Irlande, le Danemark et la Belgique.

Les échanges internationaux apparaissent fortement soutenus par les bienfaits sanitaires attribués au lait de chamelle, même s'il existe très peu d'études de marché permettant de valider cette hypothèse. L'engouement de certains consommateurs pour ce lait se répercute sur son prix significativement plus élevé que celui du lait de vache. Il ne constitue en rien un lait de substitution. Son prix et son volume de production le confinent dans une production de niche qui s'explique à la fois par des coûts de production élevés, par une consommation encore peu développée, et par l'absence de normes internationales.

Trois éléments nous paraissent essentiels à prendre en compte pour expliquer la croissance du marché du lait de chamelle : a) la richesse et l'originalité de la composition du lait qui constituent des atouts commerciaux auprès des consommateurs, b) l'offre diversifiée de produits transformés (lait aromatisé, laban, fromage, crème glacée, yaourt, poudre de lait entier et écrémé, préparations pour nourrissons, produits cosmétiques), et c) la grande diversité des circuits commerciaux mobilisés pour commercialiser ces produits (grandes et moyennes surfaces, boutiques spécialisées, réseaux des boutiques « nature » ou « bien-être », commerce en ligne, etc.). D’ailleurs, les ventes ont été stimulées par la pandémie du Covid-19 dans plusieurs régions. En effet, en se basant sur le fait que les immunoglobulines des camélidés permettent de développer des anticorps thérapeutiques (Dong et al., 2020), plusieurs experts ont évoqué un effet potentiel du lait de chamelle pour augmenter l'immunité anti-Covid-19. Ce nouvel argument commercial s'est traduit dans certains pays comme le Kazakhstan par une augmentation importante de la demande et du prix du lait de chamelle (Nagy et al., 2020). Cet engouement pour les possibles vertus immunostimulantes de ce lait a également accru le volume des exportations (Anonyme, 2019). Ainsi, la demande chinoise pour le lait de chamelle produit au Kazakhstan a augmenté de $20 \%$ pendant la crise sanitaire (www.chinadaily.com.cn/a/ 202007/06/WS5f0288dda310834817257656_3.html).

\section{- CONCLUSION}

L'analyse des sites de vente en ligne du lait de chamelle met en évidence l'émergence de nouveaux acteurs qui s'investissent dans l'économie laitière cameline. Alors que les élevages pastoraux mobiles restent dominants dans la plupart des pays d'élevage, notre étude souligne l'apparition de nouveaux entrepreneurs qui s'investissent dans des élevages plus intensifs, et qui prennent en charge le commerce et la transformation de produits transformés. Ces nouveaux entrepreneurs du lait de chamelle sont implantés pour une bonne partie dans des pays dépourvus d'élevages camelins, notamment en Europe, aux Etats-Unis, au Canada et dans plusieurs pays émergents (Brésil, Chine, Afrique du Sud).

La dynamique du commerce en ligne du lait de chamelle reflète ainsi une dynamique d'innovation duale. D'un côté, l'apparition d'unités de production intensives orientées vers un marché de longue distance rend compte de l'émergence d'une économie entrepreneuriale. Cette économie qui s'appuie notamment sur les ventes en ligne apparait relativement déconnectée des dynamiques pastorales. Elle est le fait de nouveaux investissements privés qui permettent de répondre à une nouvelle demande urbaine. Cette forme d'économie cameline de type extraterritorial témoigne de la recherche par les consommateurs de nouveaux produits identitaires, diététiques, durables ou fonctionnels. D'un autre côté, le développement d'ateliers de production laitière périurbains participe au développement d'une économie cameline de proximité. Ces ateliers sont initiés par des éleveurs pastoraux qui pratiquent un allotement des femelles et procèdent à des améliorations à la marge des pratiques d'alimentation. Ces ventes de proximité, limitées aux villes proches des zones pastorales, donnent lieu à l'émergence d'une économie beaucoup plus inclusive, c'est-à-dire fortement corrélée aux élevages familiaux et aux territoires pastoraux (Duteurtre, 2019).

Richesse pastorale par excellence, la chamelle laitière est ainsi devenue un capital productif mobilisé par les acteurs de l'économie numérique. Cette nouvelle perspective souligne que les dimensions technicoéconomiques du commerce des produits de l'élevage se doublent de dimensions plus sociales, voire sociopolitiques qu'il importe de prendre en compte (Duteurtre et Faye, 2009).

Le développement de cette économie duale pose de nouvelles questions de recherche. Il s'agit de mieux connaître les caractéristiques des laits de chamelle issus des systèmes plus intensifiés, et leurs propriétés sanitaires et nutritionnelles. L'émergence de ce nouveau commerce soulève aussi des besoins en termes de réglementation pour réguler ces ventes sur des marchés où il n'existe pas de standards de qualité. Les entrepreneurs font par ailleurs face à de nombreuses contraintes techniques pour élaborer de nouveaux produits laitiers. Il s'agit aussi d'évaluer les impacts des nouveaux investissements privés dans la filière sur les débouchés commerciaux pour les éleveurs pastoraux. La question du développement des filières camelines pose en effet la question de leur impact sur la réduction de la pauvreté. Il est urgent d'identifier des stratégies et des programmes de développement pour rendre cette nouvelle économie entrepreneuriale plus inclusive. 


\section{Remerciements}

Cet article, réalisé dans le cadre du programme Mak'IT (Camel milk technology and markets: a new frontier for sustainable food systems in the 21st century), a bénéficié du soutien du programme PRIMA de l'Union européenne dans le cadre du projet $n^{\circ} 1832$ "Boost the production, processing and consumption of camel milk in the Mediterranean Basin (Camelmilk)".

\section{Déclaration des contributions des auteurs}

GK et $\mathrm{BF}$ ont collecté les données, traité les informations et rédigé l'article en commun. GD a supervisé le projet et contribué à la rédaction finale.

\section{Conflits d'intérêts}

Les auteurs déclarent que l'étude a été réalisée sans conflit d'intérêts.

\section{REFERENCES}

Abdalla E.B., Ashmawy A.E.A., Farouk M.H., Salama O.A.E., Khalil F.A., Seioudy A.F., 2015. Milk production potential in Magrebi she-camels. Small Rumin. Res., 123: 129-135, doi: 10.1016/j.smallrumres.2014.11.004

Abeiderrahmane N., 1997. Camel milk and modern industry. J. Camel Pract. Res., 4, 223-228

Al-Abri M., Faye B., 2019. Genetic improvement in dromedary camels: challenges and opportunities. Front. Genet., 10 (167): 1-5, doi: 10.3389/ fgene.2019.00167

Anonyme. 2019. Article de press Tengrinews.kz au Kazakhstan en russe : "Kazakhstan planifie d'exporter le lait de chamelle vers la Chine " 16/09/2019, [Казахстан планирует экспортировать верблюжье молоко в Китай: 16 сентября 2019, новости на Tengrinews.kz]

Ayadi M., Musaad A., Aljumaah R., Matar A., Konuspayeva G., Abdelrahman M., Abid I., et al., 2018. Machine milking parameters for an efficient and healthy milking in dairy camels (Camelus dromedarius). J. Camel Prac. Res., 25 (1): 81-88, doi: 10.5958/2277-8934.2018.00012.7

Burger P., Ciani E., Faye B., 2019. Old world camels in a modern world - A balancing act between conservation and genetic improvement. Anim. Genet., 50: 598-612, doi: 10.1111/age.12858

Chamekh L., Calvo M., Khorchani T., Castro-Gómez P., Hammadi M., Fontecha J., Yahyaoui M.H., 2020. Impact of management system and lactation stage on fatty acid composition of camel milk. J. Food Compos. Anal., 87: 103418, doi: 10.1016/j.jfca.2020.103418

Dong J., Huang B., Jia Z., Wang B., Kankanamalage A. Titong A., Liu Y., 2020. Development of multi-specific humanized llama antibodies blocking SARSCoV-2/ACE2 interaction with high affinity and avidity. Emerg. Microb. Infect., 9 (1): 1034-1036, doi: 10.1080/22221751.2020.1768806

Duteurtre G., 2019 : « Les produits laitiers africains à l'épreuve de la libéralisation: Des traditions laitières en danger, un patrimoine à valoriser ", communication aux 3es Rencontres internationales "Lait, vecteur de développement" de Dakar, Sénégal, 12-13 juin 2019, 11 p. https://colloque. inra.fr/lait2019/content/download/3873/41610/file/DUTEURTRE_Diversit\%C3\%A9\%20des\%20produits\%20laitiers.pdf

Duteurtre G. et Faye B. (Coord.), 2009. L'élevage, richesse des pauvres : Stratégies d'éleveurs et organisations sociales face aux risques dans les pays du Sud, Quae, Versailles, France, 286 p.

Elhadi Y.A., Nyariki D.M., Wasonga O.V., 2015. Role of camel milk in pastoral livelihoods in Kenya: contribution to household diet and income. Pastoralism, 5: 1-8, doi: 10.1186/s13570-015-0028-7

FAOstat, 2021. www.fao.org/faostat/en/\#home

Faye B., 2004. Dairy productivity potential of camels. Proc. of the 34th meeting FAO/ICAR (International Committee for Animal Recording). Session on camelids. 28 mai-3 juin 2004, Sousse, Tunisie, 93-105

Faye B., 2016. The camel, new challenges for a sustainable development. Trop. Anim. Health Prod., 48: 689-692. doi: 10.1007/s11250-016-0995-8

Faye B., 2018a. The enthusiasm for camel production. Editorial. Emir. J. Food. Agric., 30 (4): 249-250
Faye B., 2018b. The improvement of the camel reproduction performances: just a technical question? Rev. Maroc. Sci. Agron. Vét., 6 (2): 265-269

Faye B., 2018c. What future for camel pastoralism in the world? Proc. The 5th Conference ISOCARD "Recent advances in camelids biology, health and Production", 12-15 Nov. 2018, Laâyoune, Morocco (A. Sghiri \& F. Kichou Eds), IAV Hassan II, 32-38

Faye B., 2020. How many large camelids in the world? A synthetic analysis of the world camel demographic changes. Pastor.: policy, res. prac., 10: 25 doi: 10.1186/s13570-020-00176-z

Faye B., Chaibou M., Vias G., 2012. Integrated impact of climate change and socioeconomic development on the evolution of camel farming systems. Brit. J Envir. Clim. Change, 2 (3): 227-244, doi: 10.9734/BJECC/2012/1548

Faye B., Jaouad J., Bhrawi K., Senoussi A., Bengoumi M., 2014b. Elevage camelin en Afrique du Nord : état des lieux et perspectives. Rev. Elev. Med. Vet. Pays Trop., 67 (4): 213-221. doi: 10.19182/remvt.20563

Faye B., Konuspayeva G., 2012b. The sustainability challenge of the dairy sector- The growing importance of the non-cattle milk production worldwide. Int. Dairy J, 24: 50-56, doi: 10.1016/j.idairyj.2011.12.011

Faye B., Konuspayeva G., 2017. The new horizons of the camel industry in the world. Proc. Intl Conf. "the belt and road camel science, industry and culture", 22-26 Sept. 2017, West Alxa, China, 13-16

Faye B., Madani H., El-Rouili A.H., 2014a. Camel milk value chain in Northern Saudi Arabia. Emir. J. Food Agric., 26 (4): 359-365, doi: 10.9755/ejfa. v26i4.17278

Koussou M.-O., 2008. Dynamique des changements dans le secteur de l'élevage au Tchad : le cas de la filière laitière de N'Djamena. Thèse de doctorat de I'Institut des Sciences et Industries du Vivant et de I'Environnement (AgroParisTech), Spécialité : Systèmes d'élevage et filières animales, Paris, $238 \mathrm{p}$.

Koussou M.-O., Mahamat Ahmat Mahamat A., Grimaud P., 2012. Campements nomades et commerce du lait de chamelle en périphérie de N'Djamena (Tchad). Rev. Sci. Tchad, 11 (1): 34-41

Nagy P., Bin Subaih S.J., Al-Badri M., Juhasz J., 2014. The "bumpy" road of camel milk approval from United Arab Emirates to the European Union: the Camelicious experience. Actes des 1res rencontres internationales sur «le lait, vecteur de développement », 21-23 mai 2014, Rennes (France), C28, 174 p.

Nagy P., Faigl V., Reiczigel J., Juhasz, J., 2015. Effect of pregnancy and embryonic mortality on milk production in dromedary camels (Camelus dromedarius). J. Dairy Sci., 98 (2): 975-986, doi: 10.3168/jds.2014-8546

Nagy P., Wernery U., Burger P., Juhasz J., Faye B., 2020. The impact of COVID-19 on Old World Camelids and their potential role to combat a human pandemic. Animal Frontiers, 11 (1) : 60-66, doi: 10.1093/af/vfaa048

Srairi T., Chatellier V., Corniaux C., Faye B., Aubron C., Hostiou N., Safa A., et al., 2019. Réflexions sur le développement du secteur laitier et sa durabilité dans différentes parties du monde. INRA Prod. Anim., 32 (3): 339-358, doi: 10.20870/productions-animales.2019.32.3.2561

Yadav A.K., Kumar R., Priyadarshini L., Singh J., 2015. Composition and medicinal properties of camel milk: A Review. Asian J. Dairy Food Res., 34 (2): 83-91, doi: 10.5958/0976-0563.2015.00018.4 


\section{Summary}

Konuspayeva G., Faye B., Duteurtre G. Online camel milk trade: new players, new markets

The rapid growth of the Dromedary and Bactrian camel population worldwide aims in part at satisfying a growing demand for camel milk. We analyze these changes by studying the recent arrival of online trade of camel milk products. This study highlights the emergence of actors involved in the intensive production of camel milk, but also in the trade of milk powder fermented milk and cheese. These new camel milk entrepreneurs are located for the most part in countries without camel farms, notably in Europe, the United States, Canada and several emerging countries. The prices of milk sold over the internet are extremely variable, highlighting a market that is under construction. The emergence of this trade reflects a dualistic innovation process. This entrepreneurial dairy economy based on exchanges between intensive livestock farms and distant consumers sharply contrasts with a local dairy economy based on peri-urban trade in fresh milk, which is strongly correlated with pastoral systems. We conclude with research and development recommendation to make the online trade of camel milk more inclusive.

Keywords: camel milk, consumption, exports, international trade, electronic commerce

\section{Resumen}

Konuspayeva G., Faye B., Duteurtre G. Comercio en línea de leche de camella: nuevos actores, nuevos mercados

El crecimiento rápido del hato mundial de dromedarios y de camellos se enfoca principalmente hacia responder a una demanda creciente de leche de camella. Analizamos estas mutaciones mediante el estudio del comercio en línea de productos originarios de la leche de camella, de aparición reciente. Este estudio realza el surgimiento de los actores involucrados en la producción intensiva de leche de camella, así como en el comercio de leche en polvo, de leche fermentada y de quesos. Estos nuevos empresarios de leche de camella se encuentran implantados, en su mayoría, en los países desprovistos de explotaciones camelinas, sobre todo en Europa, Estados Unidos, Canadá y en varios países emergentes. Los precios de la leche vendida por internet son extremadamente variables, realzando que este mercado se encuentra en curso de construcción. El surgimiento de este comercio refleja una dinámica de innovación doble. A esta economía lechera empresarial basada sobre intercambios entre fincas de cría intensiva y de consumidores distantes, se opone en efecto una economía lechera de proximidad basada sobre el comercio periurbano de leche fresca, fuertemente correlacionado a los sistemas pastoriles. Concluimos sobre las pistas de investigación y de desarrollo para hacer el comercio numérico de leche de camella más inclusivo.

Palabras clave: leche de camella, consumo, exportaciones, comercio internacional, comercio electrónico 